\title{
Characterization of dust collected after plasma operation of all tungsten ASDEX Upgrade
}

\author{
E Fortuna-Zalesna $^{\mathrm{a}, *}$, J Grzonka ${ }^{\mathrm{a}}$, M Rasinski ${ }^{\mathrm{a}}$, M Balden ${ }^{\mathrm{b}}$, V Rohde ${ }^{\mathrm{b}}$, K J \\ Kurzydlowski ${ }^{\mathrm{a}}$, ASDEX Upgrade Team ${ }^{\mathrm{b}}$ \\ ${ }^{a}$ Faculty of Materials Science and Engineering, Warsaw University of Technology, \\ Association EURATOM-IPPLM, 02-507 Warsaw, Poland \\ ${ }^{b}$ Max-Planck-Institut für Plasmaphysik, Association EURATOM, D-85748 Garching, \\ Germany \\ E-mail*:efortuna@inmat.pw.edu.pl
}

PACS: 28.52.Fa, 68.37.Ma, 68.37.Hk, 81.05.Je

\begin{abstract}
The work presents results of the characterization of dust collected in ASDEX Upgrade, with the special emphasis on size, morphology, structure and composition of the dust particles. The dust particles were collected after 2009 campaign using the filtered vacuum technique. The structure and composition of the particles were examined by scanning electron microscopy (SEM) combined with energy-dispersive X-ray spectroscopy (EDS), focused ion beam (FIB) and scanning transmission electron microscopy (STEM) with special interest on the tungsten particles.
\end{abstract}

Keywords: tokamak dust, ITER

\section{Introduction}

It is well established that dust particles, formed in magnetic confinement fusion devices as consequence of plasma-wall interactions, may influence the plasma stability. Re-deposition of dust also could reduce the optical transmission of diagnostic windows. For future fusion reactors dust may cause serious hazards connected with the release of tritium accumulated inside the plasma-facing material, e.g., by steam reactions in the case of cooling water leaks into the vessel. Thus, detailed examinations of dust particles appearing in present-day machines are needed for the operation of ITER and DEMO.

\section{Experimental}

The examinations were carried out on dust particles trapped in the membrane rims of filters no 1 and 8, collected after 2009 ASDEX Upgrade campaign (three boronization, 1101 shots and 5274s plasma), from sector 8 (above an ECRH mirror) and 11 (on tiles of Bg10), respectively (see Fig. 1). Filter no 1 represents the dust at the low field side midplane and filter 8 the outer divertor structure. The filtered vacuum cleaning was performed using Whatman Anapore 451 aluminium oxide membranes with 0.02 $\mu \mathrm{m}$ pore size [1].

The dust particles have been investigated in terms of their size/morphology, composition and structure, using scanning electron microscopy (Hitachi SU-70 FE-SEM) combined with energy-dispersive X-ray spectroscopy (EDS), focused ion beam (Dual Beam Hitachi NB5000) and scanning transmission electron microscopy (Aberration Corrected Dedicated STEM Hitachi HD-2700) techniques. 
This work was a supplement of investigations presented in [2], of automatic dust analyses done by commercial SEM-EDS system for classification of dust particles present in all tungsten ASDEXUpgrade (AUG).

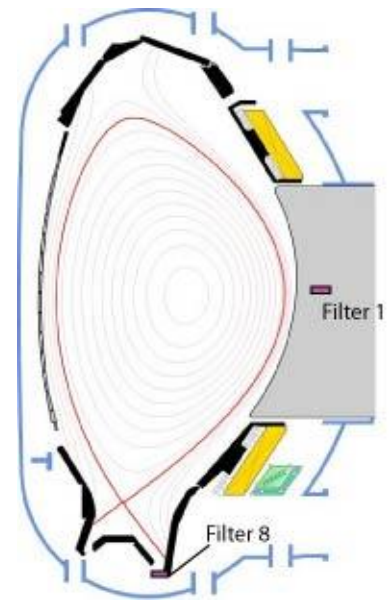

Fig. 1. Filters position in AUG

\section{Results}

\subsection{Outer morphology and elemental composition}

Representative SEM images of the dust particles are shown in Figs. 2-4. It has been found in observations that on filter 1, carbon based particles appear in the largest number (85\% of examined population) with iron, tungsten and copper based particles in minority. In the case of filter 8 the largest fraction comprises tungsten dust (60\% of examined population). The second largest group are carbon based particles (30\% of examined population). The iron particles were also found.

The carbon based particles are mainly small fragments of co-deposited layers $(\mathrm{C}+\mathrm{O}+\mathrm{Si}+\mathrm{Fe}+\mathrm{Cr}$, size: 2-23 $\mu \mathrm{m}$ ). Other particles found are probably small parts of CFC and graphite tiles (size: up to 300 $\mu \mathrm{m})$. The iron-based particles included small metallic chips and flakes (size: 1-25 $\mu \mathrm{m}$ ). In addition to iron they contain chromium and nickel, typical components of stainless steel (SS301), which is used for the wall vacuum vessel and the supporting structure of the plasma facing components, especially the tungsten-coated graphite tiles. In the dust particles boron was also detected. The crystals rich in boron were present in part of tungsten based particles. It was also very likely present in part of carbonbased particles.

Filter no 1 is typical for all dust mobilized at the low field side of AUG. There the structure is quite complicated and many deposition dominated areas exists. The carbon flakes may come from these areas. Filter no 8 represents the outer divertor structure. The Bgr 10 is the horizontal plane just below the divertor plates, collecting all dust falling down from the outer divertor, which is a net erosion dominated region.

Since 2007 ASDEX-Upgrade is a full tungsten machine, nearly all plasma-facing surfaces are covered with tungsten [3-5]. The tungsten particles were thus of a special interest in the present study. Based on the results of SEM investigations they can be classified into the following types:

a) spherical particles $(1$ to $7 \mu \mathrm{m})$ with characteristic shell and core structure, which account for $33 \%$ of the examined population

b) irregularly-shaped particles ( 1 to $20 \mu \mathrm{m}$ ), of columnar or multilayer structure, $32 \%$ of the examined population

c) flakes (up to $70 \mu \mathrm{m}$ ) with columnar structure, $28 \%$ of the examined population

d) small particles at/inside the large carbon and iron-based ones, $7 \%$ of the examined population

In order to infer the most probable origin of these particles their detailed structure examinations were carried out with the results presented in the following sections.

\subsection{Inner morphology examinations on cross-sections}


The internal structures of tungsten based particles were investigated via observations of 88 crosssections, which have been prepared by FIB. The same types of particles were present on both filters. In the case of the spherical particles three types of microstructures were revealed, Fig. 2: (a) grained, (b) two phased, with one tungsten or tungsten rich and the other containing boron and carbon $(15 \%$ of the sectioned spherical particles) and (c) of percolated morphology (6\% of the sectioned spherical particles).
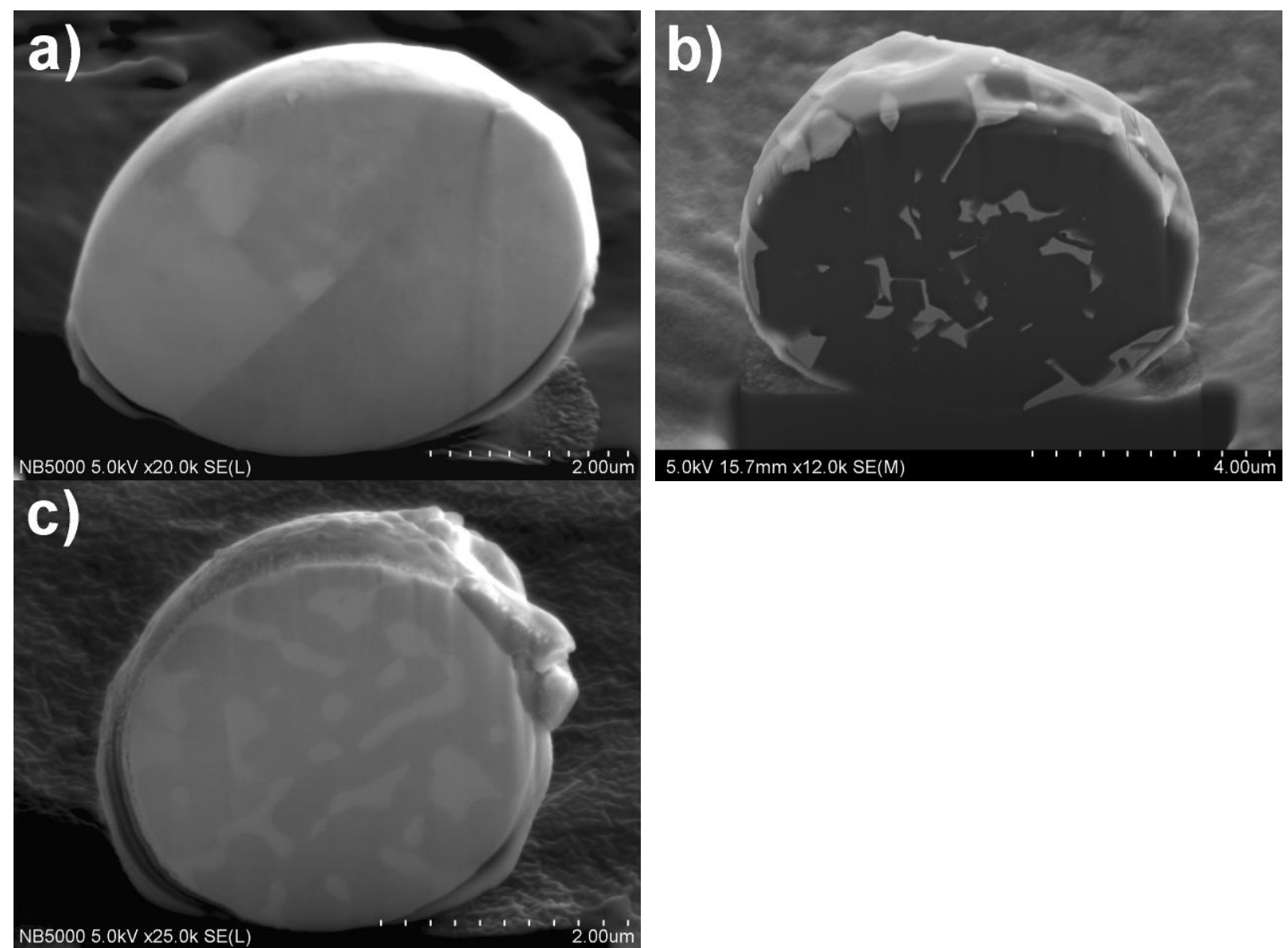

Fig. 2. SEM images of cross-sections of spherical tungsten particles: a) grained structure, b) two phased, c) with percolated morphology

The particles of irregular shape, shown in Fig. 3 can be classified into three types of microstructures. Some of them are fine-grained, Fig. 3a, some contain small grains rich in boron (dark crystals in Fig. $3 b$ ) and white ones rich in tungsten (25\% of the sectioned irregular particles). At the cross-sections of other particles small grains and considerable porosity were revealed, Fig. $3 \mathrm{c}$ (12\% of the sectioned irregular particles). 

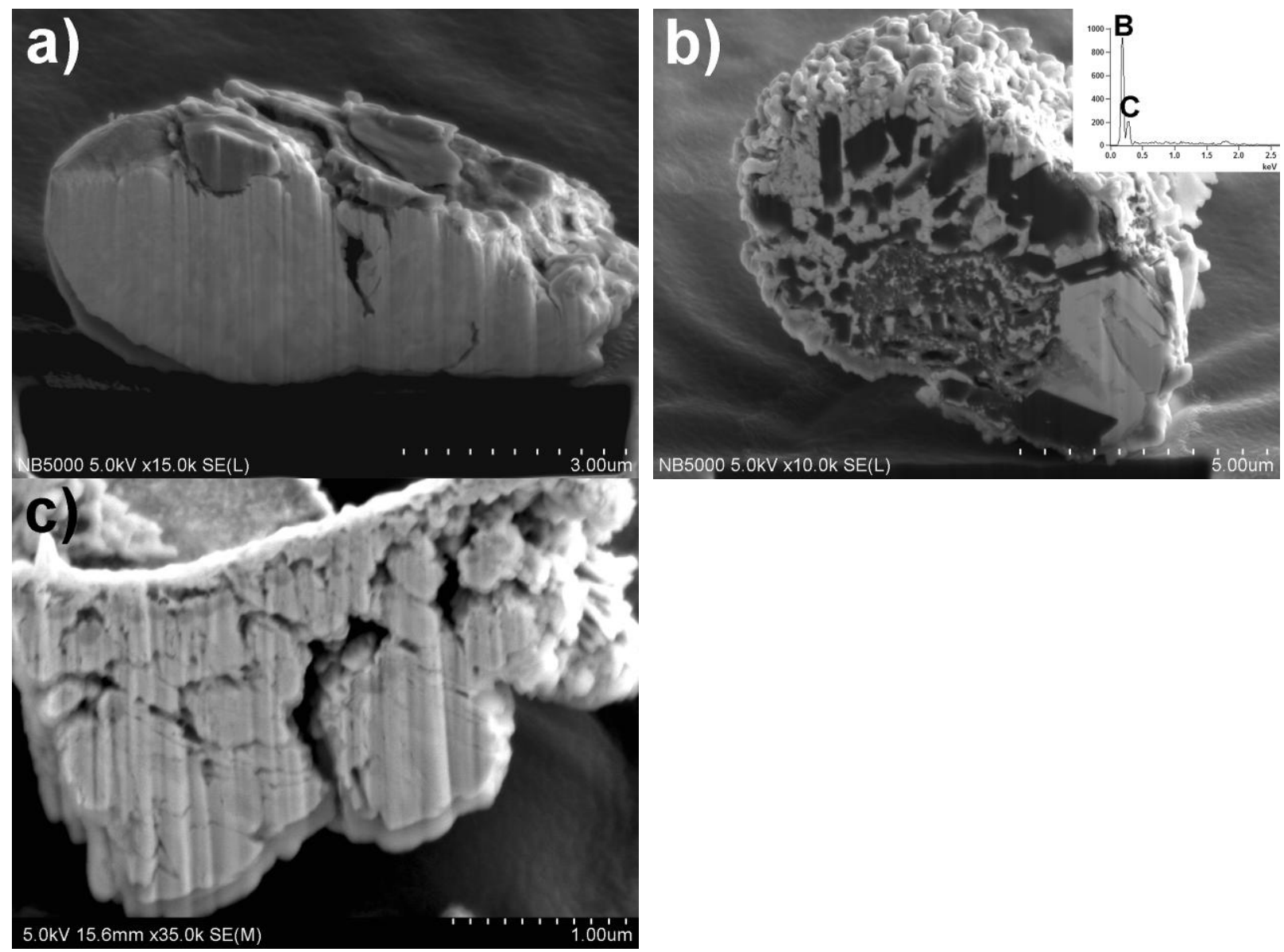

Fig. 3. SEM images of cross-sections of tungsten-based particles of irregular shape: a) fine-grained, b) two phased, c) porous with small grains

The cross-sections of tungsten flakes presented in Fig. 4 illustrate their columnar character, characteristic for the PVD tungsten coatings in ASDEX Upgrade.
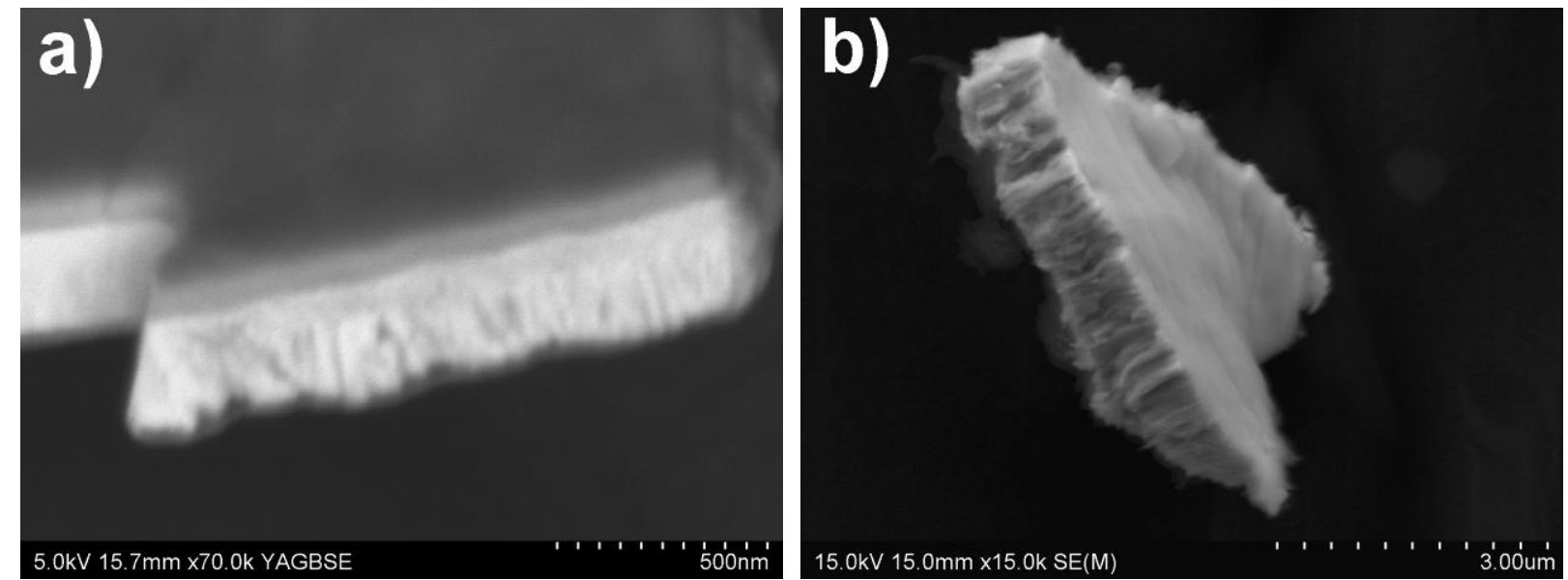

Fig. 4. SEM images of thin flakes

\subsection{Internal structure examinations on thin foils}

TEM image of the fine-grained particle is presented in Fig. 5a. Small, elongated grains (100-300 nm thick) arranged into a columnar structure with characteristic dislocation walls are observed. It should be noted that similar structure has been observed in a $4 \mu \mathrm{m}$ tungsten PVD layer of Bg 04/1 tile from the inner strike point region working in AUG during 2007 campaign.

The structure of a particle with boron rich phase is presented in Fig. 5b. The dark phase was identified by diffraction patterns as boron carbide. Further studies are carried out to identify bright crystals. The 
fact that they do not reveal artefacts induced by ion milling suggests that it is not pure tungsten but its intermetallic phase/phases. (The structure of particles with grains rich in boron is the same as the deposited layers at the inner divertor baffle region).

In the case of the particle with percolated morphology, the EDS measurements at the TEM thin foil reveal no difference in the chemical composition. The details and origin of such a structure is not known yet.
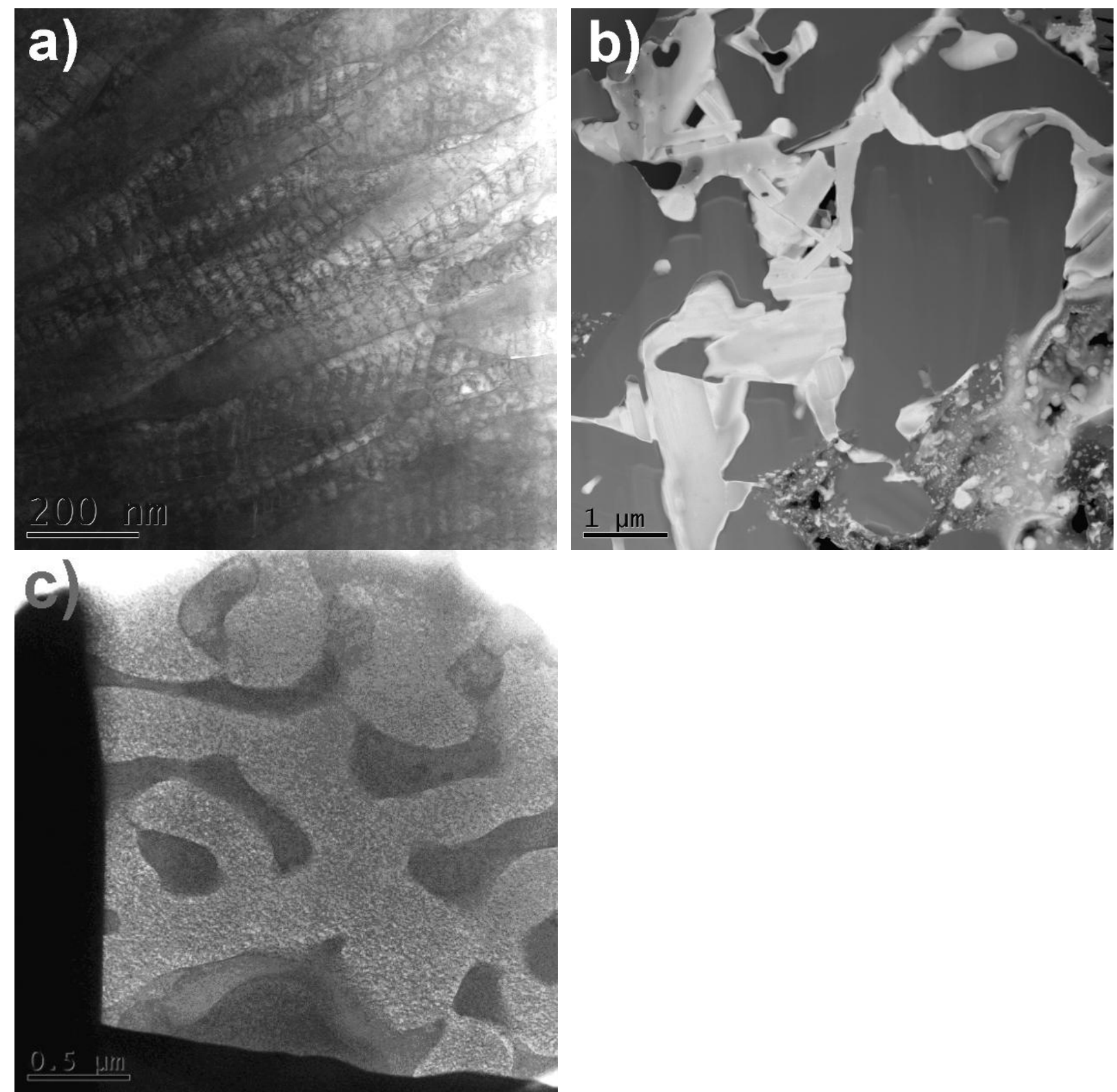

Fig. 5. TEM images of: a) fine-crystalline particle, b) a particle with two phases, c) a particle with percolated morphology

\section{Discussion}

Characterization of the morphology and measurements of chemical composition of dust particles could link the particles to the most probable production site. During the 2009 campaign dust has been trapped on five silicon wafer collectors strategically positioned within the vacuum vessel of the full tungsten ASDEX-Upgrade [2] (see also [3-4] for previous examinations). The automated SEM-EDS was subsequently used to examine the morphology and chemical composition of $5 \times 10^{4}$ particles, population of which turned out to contain tungsten, carbon, boron, iron and copper based ones. Except for one collector, the tungsten particles dominated by accounting for $50-65 \%$ of the examined sample. 
The present study was performed to extend this investigation by detailed examinations of internal structure of tungsten based particles.

In discussion of the results obtained it should be noted first that there is a large diversity in morphologies and internal structures of tungsten-based particles. As far as morphology is concerned the particles can be categorised into three classes: spherical, irregular and flakes, each about 1/3 of the total population. The present results clearly show that these spherical particles ( 1 to $7 \mu \mathrm{m})$ profoundly differ in their structures, which can be broadly classified as: grainy, two phased, (with one tungsten or tungsten rich and the other containing boron) and percolated. The irregularly-shaped particles (1 to 20 $\mu \mathrm{m})$ are primarily of columnar structure and most likely come from co-deposited layers and tungsten coating. In these group the particles with grains rich in boron were also present. The analyses carried out at the particle containing tungsten, boron and carbon prove that the intermetallic phases both carbides and borides are present in the dust particles/deposited layers [5,6]. The tungsten flakes are predominantly fragments of tungsten layers.

One should take into account that morphology and size of dust particles could change during their movements in the machine. Thus the knowledge of their internal structure and composition provides important information on their origin. The short discussion concerning possible dust production sites and relevant processes of dust formation is presented below.

Since 2007 AUG operates as a full tungsten machine [7-9]. In 2009 campaign two kinds of tungsten coatings were present: (i) physical vapour deposited (PVD) coatings with thickness up to $4 \mu \mathrm{m}$ in the main chamber, the inner strike line module and the upper divertor and (ii) a $10 \mu \mathrm{m}$ CMSII tungsten coating (combined magnetron sputtering and ion implantation technique) on $3 \mu \mathrm{m}$ Mo interlayer [10] in the outer divertor.

The main dust production mechanism in AUG seem to be arcing $[4,11,12,13]$. Other tungsten sources such as molten layers of coatings have to be taken into account [14]. The third possible dust production mechanism is coating delamination due to thermal stresses during the plasma operation [8]. From investigations of V. Rohde et al [13] one can find out that in total about $2 \mathrm{~g}$ of tungsten was eroded by arcing during the 2009 campaign, with a strong erosion observed at the inner baffle region. This is about $10 \%$ of the net physical sputtering in the outer divertor region [13, 15]. Local erosion by arcing can destroy the tungsten coating of the tiles [13]. It should be also noted that the arcs produce a significant amount of droplets and flakes. Spherical tungsten dust particles found in our examinations are interpreted as droplets released during arcing. Concerning the tungsten particles rich in boron, their morphology is the same as the deposited layers at the inner divertor baffle region, close to the arcs tracks (fractionised/destroyed by arcing).

The structure of the dust particle with fine elongated grains (Fig. 5a) is the same as the structure of a PVD coating. This observation as well as the columnar character of examined flakes indicate that part of the dust particles are small fragments of tungsten coatings (delamination/abrasion).

\section{Acknowledgement}

This work was supported by EURATOM and carried out within the framework of the European Fusion Development Agreement. The views and opinions expressed herein do not necessarily reflect those of the European Commission.

\section{References}

[1] Sharpe JP et al 2003, J. Nucl. Mater. 313-316 455

[2] Endstrasser N et al 2011, J. Nucl. Mater. 415 S1085

[3] Endstrasser N et al 2011, Phys. Scr. T14 014021

[4] Rohde V et at 2009, Phys. Scr. T138 014024

[5] Fortuna E et al 2007, Phys. Scr. T128 162-165

[6] Rasinski M, Ph. D thesis, Warsaw University of Technology 2012

[7] Neu R et at 2009, Phys. Scr. T138 014038

[8] Herrmann A et at 2009, Phys. Scr. T138 014059

[9] Herrmann A et at 2011, Phys. Scr. T145 014068

[10] Ruset C et at 2007, Phys. Scr. T128 171

[11] Rohde V et al 2011 J. Nucl. Mater. 415 S46

[12] Herrmann A et at 2009, J. Nucl. Mater. 390-391 747 
[13] Rohde V et al 2013 J. Nucl. Mater. 438 S800

[14] Coenen J W et al 2011 J. Nucl. Mater. 415 S78

[15] Mayer M et al 2009 Phys. Scr. T138 014039 